\title{
Complications of Varicella - Report of Case with Hemorrhagic-Necrotic Rash and Cerebellar Ataxia
}

\author{
Galya Ivanova Gancheva ${ }^{1}$, Tsetsa Georgieva Doichinova ${ }^{1}$ and \\ Tsvetan Christoforov Lukanov ${ }^{2}$ \\ ${ }^{1}$ Department of Infectious Diseases, Epidemiology, Parasitology and Tropical medicine - Medical \\ University, Pleven, Bulgaria \\ ${ }^{2}$ Department of Clinical laboratory, Clinical Immunology and Allergology - Medical University, Pleven, \\ Bulgaria
}

Correspondence should be addressed to: Galya Ivanova Gancheva; galya_gancheva@abv.bg

Received Date: 20 November 2013; Accepted Date: 23 March 2014; Published Date: 29 April 2014

Academic Editor: Roman Chlíbek

Copyright (C) 2014 Galya Ivanova Gancheva, Tsetsa Georgieva Doichinova and Tsvetan Christoforov Lukanov. Distributed under Creative Commons CC-BY 3.0

\begin{abstract}
Varicella is usually self-limiting but occasionally severe infectious disease (with 2 to $6 \%$ of cases resulting in complications). The aim was to report a case with hemorrhagic-necrotic rash, discrete cerebellar ataxia and immune suppression. A two-year-and-seven-month-old boy with fever, tonsillitis and rose-colored-spots rash was treated with Amoxiclav and antihistamines followed by improvement. Eight days after treatment, fever, vesiculous rash (consequently hemorrhagicnecrotic) and enlarged lymph nodes appeared. On admission, the child was in severe condition, with generalized polymorph rash (vesicles with bloody content on necrotic surface, few crusts), generalized enlarged and painful lymph nodes, increased breath and heart rates, hepatosplenomegaly, depressed patellar and Achilles reflexes, positive Babinski sign; without signs of meningeal irritation. Ataxia had observed and Romberg was positive. Laboratory investigations revealed anemia, thrombocytopenia and normal humoral immunity. Lymphopenia (12.28\%), decreased total T-lymphocytes, normal CD4+/ CD8+ T-lymphocytes ratio at normal CD4+ and decreased CD8+ T cells, increased B-lymphocytes were found by flow-cytometry. Serological investigations (ELISA) revealed significant titers of specific IgM antibodies against three different herpesviruses (HSV-1, HSV-2 and Varicella zoster virus). Complex etiologic and supportive treatment was administered. The child had improved after sixth day and was discharged on eleventh day. Hemorrhagic-necrotic form of varicella is rare. It occurs mainly in immunocompromised patients, but immunocompetent individuals also could be affected. This requires the use of broad diagnostic panel for precise etiological diagnosis, which will facilitate the proper treatment and favorable outcome.
\end{abstract}

Keywords: Varicella, cerebellar ataxia, thrombocytopenia, immunosupresion.

Cite this Article as: Galya Ivanova Gancheva, Tsetsa Georgieva Doichinova and Tsvetan Christoforov Lukanov (2014), "Complications of Varicella - Report of Case with Hemorrhagic-Necrotic Rash and Cerebellar Ataxia," JMED Research Vol. 2014 (2014), Article ID 589754, DOI: 10.5171/2014.589754 


\section{Introduction}

Varicella is a common infectious disease with high susceptibility. As Bonanni et al. (2009) noted, although the disease is usually self-limiting, some cases of varicella can be serious, and complications have been observed in 2 to $6 \%$ of cases attending a general practice. According to the same authors, the hospitalization rate for varicella in Europe ranges from 1.3 to 4.5 per 100000 population/year and up to $10.1 \%$ of hospitalized patients report permanent sequels (for example, scarring or ataxia).

Varicella is caused by the varicella zoster virus (VZV), a member of the alphaherpesvirus subfamily. VZV replication is highly cell-associated. In immunocompetent individuals with varicella, only 0.01 to $0.001 \%$ of peripheral blood mononuclear cells (PBMCs) contain $\mathrm{VZV}$. As was quoted in the review of Arvin (1996), it has been difficult to identify the PBMCs subpopulations that become infected because of the low frequency of positive cells. Lymphocytes and monocyte/macrophage cell types may harbor VZV and the virus is lymphotropic for CD4+ and CD8+ T-lymphocytes. VZV spreads to epidermal cells by cell-associated viremia, and replication in these cells causes the typical rash. Usually superficial epithelial cells are infected and a damage of the germinal layer leads to permanent scars (Arvin, 1996).

VZV has the potential to cause disseminated infection in immune compromised individuals. Cerebellar ataxia is the most common neurologic complications of varicella. Encephalitis may be caused by direct spread of VZV inducing vasculitis. Other possibility is that some central nervous system manifestations (CNS) of varicella are provoked by immune system mediated damage (Arvin, 1996). In research study of Fritzler et al. (2003) it was shown that children with cerebellar ataxia have unique autoantibody reactivity to pericentrin - a structural protein located in axons and centrosomes of cerebellar cells.

Quoted in Arvin (1996), other pathologic complications of varicella include thrombocytopenia and necrosis of the adrenal glands (in fulminant varicella). Glomerulonephritis, viral arthritis, uveitis, retinal necrosis, myocarditis, pancreatitis, and orchitis are uncommon.

We report a child with primary varicella infection manifested with hemorrhagic, necrotizing rash, discrete cerebellar ataxia and immune suppression.

\section{Case Report}

A two-year-and-seven-month-old male patient was born normally, from first normal pregnancy of the mother, with birth body weight $3,700 \mathrm{~g}$, gained normally and was without any previous health problems. On $10^{\text {th }}$ July 2013 he became sick with simultaneous fever to $39^{\circ} \mathrm{C}$, tonsillitis and rose-colored-spots rash. He was treated from his family doctor with amoxiclav and antihistamines (eight-day course of treatment, initiated on the second day after the onset of the disease). On the fourth day rash disappeared, body temperature gradually decreased to normal and the patient' state had improved. Eight days later (on $26^{\text {th }}$ July), the body temperature raised up to $39.5^{\circ} \mathrm{C}$ and vesiculous rash on the trunk, face and scalp appeared, followed by bilaterally enlargement of cervical and inguinal lymph nodes. Two days later (on $28^{\text {th }}$ July), the mother observed that the vesicles are with bloody content, surrounded by marked bluish zone. Treatment with ceftriaxon was initiated, but because of worsening, the child was hospitalized on $29^{\text {th }}$ July in Ward of Infectious Diseases at the regional hospital. The history revealed recent contact (after improving from the first illness) with neighbor child with varicella. The child was somnolent, with unstable walking. Due to rapid worsened condition, the child had been referred on $30^{\text {th }}$ July to 
our unit (Clinic of Infectious Diseases at University Hospital - Pleven).

On admission in Clinic of Infectious Diseases - Pleven (on 30th July), the child was in severe condition, febrile, somnolent. The skin examination had demonstrated a diffuse polymorph rash (vesicles with bloody content on necrotic surface and few crusts (Figure 1). The remainder physical examination revealed markedly enlarged and painful cervical, axillar and inguinal lymph nodes, increased breath and heart rates, hepatosplenomegaly, depressed patellar and Achilles reflexes, positive Babinski sign. Signs of meningeal irritation had not been found - stiff neck, Brudzinski' and Kernig' signs were negative. Cranial nerves were intact. Romberg was positive.

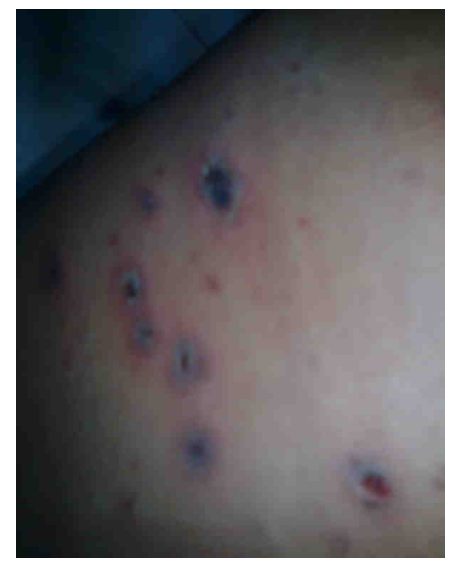

Figure 1: Hemorrhagic-Necrotic Rash in the Reported Case of Varicella

Laboratory investigations revealed leucopenia, anemia, thrombocytopenia, hypoproteinemia and hypoalbuminemia
(Table 1). Liver functional tests, electrolytes and nitrogen parameters were normal.

Table 1: Laboratory Investigations of the Reported Case after Admission in Clinic of Infectious Diseases at University Hospital - Pleven

\begin{tabular}{|l|c|c|c|c|c|c|}
\hline \multicolumn{1}{|c|}{ Laboratory parameters } & $\mathbf{1}^{\text {st }}$ day & $\mathbf{2}^{\text {nd }}$ day & $\mathbf{4}^{\text {th }}$ day & $\mathbf{8}^{\text {th }}$ day & $\mathbf{1 1}^{\text {th }}$ day & Reference \\
\hline Hemoglobin (g/L) & 110 & 94 & 79 & 85 & 101 & $120-180$ \\
\hline Erythrocytes (cells x 1012/L) & 3.71 & 3.36 & 2.89 & 3.1 & 3.37 & $3.70-5.90$ \\
\hline Hematocrit & 0.30 & 0.28 & 0.24 & 0.26 & 0.28 & $0.360-0.530$ \\
\hline MCV & 82 & 84 & 82 & 83 & 85 & $82-96$ \\
\hline Leucocytes (cells x 109/L) & 3,71 & 3.36 & 2.89 & 3.1 & 3.37 & $3.5-10.5$ \\
\hline Granulocytes (\%) & 72 & 73 & 66 & 37.9 & 32.4 & $40-70$ \\
\hline Lymphocytes (\%) & 14 & 20 & 25 & 52 & 54 & $20-48$ \\
\hline Monocytes (\%) & 14 & 9 & 8 & 10 & 13 & $1-11$ \\
\hline Platelets (cells x 109/L) & 141 & 129 & 274 & 471 & 694 & $150-400$ \\
\hline Total protein (g/L) & 44.69 & - & - & - & 60 & $64-83$ \\
\hline Albumins (g/L) & 19.8 & - & - & - & 29 & $35-50$ \\
\hline Glucose (mmol /L) & 7.78 & - & - & - & 5.1 & $3.89-6.11$ \\
\hline C-reactive protein (mg/L) & 123 & - & - & - & 16 & $0-5.00$ \\
\hline
\end{tabular}

Humoral immunity was in reference ranges IgM $194 \mathrm{mg} / \mathrm{dL}$ (N 40-230), IgG $762 \mathrm{mg} / \mathrm{dL}$ (N 700-1600), IgA $112 \mathrm{mg} / \mathrm{dL}(\mathrm{N} \mathrm{70-400).}$
Immuno-phenotypization of lymphocytessubsets, performed by FACSort flowcytometer, revealed lymphopenia $(12.28 \%$; 
N 38-53\%), decreased total T-lymphocytes (CD3+) (55.06\%; N 62-69\%); normal Thelpers/cytotoxic T cells ratio (CD4+/CD8+) (1.6; N 1.0 - 1.6) with normal T-helpers (CD4+) (33.92; N 30-40\%) and decreased cytotoxic T cells (CD8+) (20.08\%; N 25$32 \%)$, increased B-lymphocytes (CD19+) (35.86\%; N 21-28\%), normal NK-cells (CD3+/CD56+16+) (9.08\%; N 8-15\%). The interpretation of immunological investigations concluded that disorders of cellular immunity had presented.

Serological investigations (immunoenzyme method ELISA) were performed on the tenth day after admission and the results were as follow: anti-HSV-1-IgM - 31 NovaTec Units (NTU) (by validated method VLM/B8/06/2001; gray range 9-11 NTU); anti-HSV-2-IgM - 32 NTU (by validated method VLM/B8/07/2001; gray range 9-11 NTU) and significant titer of anti-VZV-IgM 47 NTU (by validated method VLM/B9/07/2001; gray range 9-11 NTU).

Treatment was performed by acyclovir (seven days), meropenem (nine days), metronidazol (tree days), glucose and saline solutions, mannitol 10\% (five days), immunovenin (trice), human albumin 20\% (twice), plasma (twice), vitamins, isoprinosine, antipyretics and antiseptic powder. During the first three days in the Clinic, the child was in very severe condition - febrile to $38.6^{\circ} \mathrm{C}$, somnolent, with inability to walk. All rash units were hemorrhagic vesicles on the necrotic surface with diameter to 7-8 $\mathrm{mm}$. The body temperature was normalized since fifth day and the child had improved after the sixth day in our Clinic - the rash had crusted, neurological examination had normalized and laboratory parameters had improved. $\mathrm{He}$ was discharged on the eleventh day after admission with normal vital functions, but some skin lesions had leaved residual scars. Instructions for continuous treatment with isoprinosine and vitamins were given. A control investigation was not made due to the departure of the child'family from the country.

\section{Discussion}

Varicella is a highly contagious and almost universal; an estimated 60 million cases occur worldwide each year. Ziebold et al. (2001) noted that varicella is benign disease of childhood, but in immunocompromised individuals the disease is severe. Complications of varicella requiring hospitalization in children are becoming more frequent in recent years than previously thought. Almuneef et al. (2006) considered that the predominance of uncomplicated cases confounds the morbidity and mortality associated with severe varicella.

According to the review of Arvin (1996) the most common cause of varicella-related morbidity in previously healthy children is secondary bacterial infection of skin lesions followed by soft tissue infections and pneumonia. In the present case only few pustulized skin lesions were observed but more of rash units were vesicles with hemorrhagic fluid.

Arvin (1996) noted that neurologic complications are the second most frequent indication for hospitalization of immunocompetent children with varicella. Meningoencephalitis and cerebellar ataxia are the major clinical manifestations of CNSinvolvement. These complications are most common in patients younger than 5 years and older than 20 years. Cerebellar ataxia has benign prognosis. The same author mentioned that transverse myelitis, causing paraplegia and sensory deficits, and transient hemiplegia due to cerebral vasculitis are rare complications. In the present case, there was only ataxia, which lasted five days and completely resolved after treatment. This allows us to assume that it is a transient effect to the cerebellum. Lack of signs of meningeal irritation and severe diffuse widespread hemorrhagicnecrotic rash did not give us reason to realize lumbar puncture and computed tomography (CT), much less nuclear magnetic resonance (NMR). 
In research study Dinleyici et al. (2012) mentioned that hemorrhagic complications of varicella are rare in otherwise healthy children with varicella, but adults are at higher risk. In review of Arvin (1996) it was shown that thrombocytopenia during acute varicella is associated with bleeding into skin lesions and visceral hemorrhage and a risk for progression to disseminated intravascular coagulopathy is possible. Except of these, purpura fulminans (caused by arterial thrombosis) is a very rare but life-threatening complication. Arvin (1996) and Mantadakis et al. (2011) considered that the main mechanism implicated in the pathogenesis of thrombocytopenia is immune-mediated platelet destruction but decreased bone marrow production of platelets, disseminated intravascular coagulation and virally-induced platelet aggregation (followed by phagocytosis or lysis) also have significance. Josephson et al. (2001) established that acute VZV infection predisposes children to a risk but nonspecific immunologic response with multiple antiphospholipid and coagulation autoantibodies (varicella-autoantibody syndrome). Increasing of B-lymphocytes in our case correlates with significant titers of specific IgM antibodies against three different herpesviruses. Mantadakis et al. (2011) mentioned that thrombocytopenia usually occurs in severe varicella, but thrombocytopenia in healthy children is without sequelae. In the present case, independent of severity, thrombocytopenia was transient and only hemorrhagic rash was observed without visceral bleeding.

Regarding treatment of varicella-induced thrombocytopenia, Mantadakis et al. (2011) considered that corticosteroids and intravenous-immunoglobulin can be used with a notice that visceral varicella could follow an administration of corticosteroids during the incubation period of varicella. We infused intravenous-immunoglobulin in dose $15 \mathrm{~mL}(1 \mathrm{ml} / \mathrm{kg})$ in three consecutive days.

Viral serology proves helpful in confirmation of diagnosis especially in cases of VZV- associated complications (Nandeesh et al., 2009). Significant titers of specific anti-HSV1-IgM, anti-HSV-2-IgM and anti-VZV-IgM were found in the present case. Hemorrhagic-necrotic form of varicella is rare and diagnostics is essential. Appearance of rose-colored-spots rash simultaneously with the rise of temperature and angina, and the resolution of rash on the background of treatment with amoksiklav dismissed the possibility for case of EBV-infection. Severe condition of the child, diffuse hemorrhagicnecrotic rash (including the scalp) and neurological symptoms gave us reason to reject the possible diagnosis impetigo contagiosa. As data were missing for aggravated premorbid status and preliminary immune deficiency, it could be assumed that the simultaneous attack by three members of the family of herpes viruses had resulted in mild immunosuppression, but it was not premorbid. As it is difficult to isolate VZV from tissues associated with the complications of varicella, a misidentification of the disease is possible. In such cases, viral serology is helpful for correct diagnosis. Serological investigation conducted to confirm the etiological diagnosis in our case, revealed the existence of significant titers of specific anti-HSV-1IgM and anti-HSV-2-IgM, and high titer of anti-VZV-IgM. According to Argirova et al. (2006) the presence of specific IgM antibodies has high diagnostic value as the high titer of these antibodies excludes a possibility for cross-reactivity. However, a later investigation of IgM antibodies is not helpful, as they rapidly become negative. According to this author IgG antibodies have not diagnostic value, even could be misinterpreted. We think that the positive result of serological investigation correlates with the clinical manifestations and confirms the diagnosis"varicella". Simultaneous presence of three herpesviruses is a possible explanation for severe course of the disease. Jarosinski (2012) experimentally confirmed that simultaneous infection of epithelial skin cells by two alphaherpesviruses occurs in the natural host. Unfortunately, due to the 
departure of the child from the country, case had not been followed clinically and serologically.

\section{Conclusion}

Hemorrhagic-necrotic form of varicella is rare. It occurs mainly in immunocompromised patients, but immunocompetent individuals also could be affected. This requires using of broad diagnostic panel for precise etiological diagnosis, which will facilitate the proper treatment and favorable outcome.

\section{References}

Almuneef, M., Memish, Z. A., Balkhy, H. H., Alotaibi, B. \& Helmy, M. (2006). “Chickenpox Complications in Saudi Arabia: Is it Time for Routine Varicella Vaccination?," International Journal of Infectious Diseases, 10:156-61.

Argirova, R. (2006). 'Diagnostic of Viral Infections,' In: Clinical Virology, edited by Dundarov S., Sofia, Medicine and Physical Culture Press, Sofia, Bulgaria.

Arvin, A. M. (1996). "Varicella-Zoster Virus," Clinical Microbiology Review, 9(3):361-381.

Bonanni, P., Breuer, J., Gershon, A., Gershon, M., Hryniewicz, W., Papaevangelou, V., Rentier, B., Rümke, H., Sadzot-Delvaux, C., Senterre, J., Weil-Olivier, C. \& Wutzler, P. (2009) "Varicella Vaccination in Europe Taking the Practical Approach," BMC Medicine 7:26. doi: 10.1186/1741-7015-726.

Dinleyici, E. C., Kurugol, Z., Turel, O., Hatipoglu, N., Devrim, I., Agin, H., Gunay, I., Yasa, O., Erguven, M., Bayram, N., Kizildemir, A., Alhan, E., Kocabas, E., Tezer, H., Aykan, H. H., Dalgic, N., Kilic, B., Sensoy, G., Belet, N., Kulcu, N. U., Say, A., Tas, M. A., Ciftci, E., Ince, E., Ozdemir, H., Emiroglu, M., Odabas, D., Yargic, Z. A., Nuhoglu, C., Carman, K. B., Celebi, S., Hacimustafaoglu, M., Elevli, M., Ekici, Z., Celik, U., Kondolot, M., Ozturk, M.,
Tapisiz, A., Ozen, M., Tepeli, H., Parlakay, A., Kara, A., Somer, A., Caliskan, B., Velipasalioglu, S., Oncel, S., Arisoy, E. S., Guler, E., Dalkiran, T., Aygun, D. \& Akarsu, S.; VARICOMP Study Group. (2012). "The Epidemiology and Economic Impact of Varicella-related Hospitalizations in Turkey from 2008 to 2010: A Nationwide Survey during the Pre-Vaccine Era (VARICOMP Study)," European Journal of Pediatrics, 171(5):817-25.

Fritzler, M. J., Zhang, M., Stinton, L. \& Rattner, J. (2003). "Spectrum of Centrosome Autoantibodies in Childhood Varicella and Post-Varicella Acute Cerebellar Ataxia," BMC Pediatrics, 3:11.

Jarosinski, K. W. (2012). "Dual Infection and Superinfection Inhibition of Epithelial Skin Cells by Two Alphaherpesviruses Co-Occur in the Natural Host," PLoS One, 7(5):e37428.

Josephson, C., Nuss, R., Jacobson, L., Hacker, M. R., Murphy, J., Weinberg, A. \& MancoJohnson, M. J. (2001). "The VaricellaAutoantibody Syndrome," Pediatric Research, 50:345-352.

Mantadakis, E., Thomaidis, S. \& Chatzimichael, A. (2011). "ChickenpoxAssociated Immune Thrombocytopenic Purpura," Hippokratia, 15(2):190.

Nandeesh, B. N., Mahadevan, A., Yasha, T. C. \& Shankar, S. K. (2009). "Hemorrhagic Pericarditis in a Child with Primary Varicella Infection (Chickenpox)," Indian Journal of Pathology \& Microbiology, 52(2):237-9.

Ziebold, C., von Kries, R., Lang, R., Weigl, J. \& Schmitt, H. J. (2001). "Severe Complications of Varicella in Previously Healthy Children in Germany: A 1-Year Survey," Pediatrics, 108(5): E79. Erratum in: Pediatrics. 2004 May 5; 113(5):1470. 